\title{
Roles of Titin in the Structure and Elasticity of the Sarcomere
}

\author{
Larissa Tskhovrebova and John Trinick \\ Institute for Molecular and Cellular Biology and Astbury Centre for Structural Molecular Biology, University of Leeds, \\ Leeds LS2 9JT, UK \\ Correspondence should be addressed to John Trinick, j.trinick@leeds.ac.uk
}

Received 16 February 2010; Accepted 13 May 2010

Academic Editor: Aikaterini Kontrogianni-Konstantopoulos

Copyright (C) 2010 L. Tskhovrebova and J. Trinick. This is an open access article distributed under the Creative Commons Attribution License, which permits unrestricted use, distribution, and reproduction in any medium, provided the original work is properly cited.

\begin{abstract}
The giant protein titin is thought to play major roles in the assembly and function of muscle sarcomeres. Structural details, such as widths of Z- and M-lines and periodicities in the thick filaments, correlate with the substructure in the respective regions of the titin molecule. Sarcomere rest length, its operating range of lengths, and passive elastic properties are also directly controlled by the properties of titin. Here we review some recent titin data and discuss its implications for sarcomere architecture and elasticity.
\end{abstract}

\section{Introduction}

The complex but extremely ordered structure of the sarcomere is the elemental force-producing machinery of striated muscles. Recent studies of sarcomere assembly $[1,2]$, protein turnover $[1,3]$, and signalling cascades $[4,5]$ provide new insights into the spectrum of intermolecular interactions that support sarcomere structure and function. There is increasing evidence that many of sarcomere properties involve the giant protein titin [2, 6-10].

The titin molecule is more than one micrometer long and in situ spans half the sarcomere, with the N-terminus in the Z-line and the C-terminus in the M-line (Figure 1(a)) [11-13]. Different isoforms (MW 3.0-3.7 MDa [14]) vary in the size and structure of the elastic I-band part of the molecule, which connects the end of the thick filament to the Z-line, as well as in the Z- and M-line regions. The size and structure of the thick filament part of titin is conserved, which is consistent with the conserved structure of thick filaments in vertebrates. Sequence shows that titin consists mainly of about 300 domains similar to immunoglobulins (Ig, I-set) and fibronectins (Fn, type-3). The elastic I-band part consists mainly of Ig domains arranged in tandem. Near the N2-line in the I-band, this arrangement is interrupted by unique sequences that bridge the "proximal" and "distal" (to the Z-line) tandem-Ig segments. In contrast, the thick filament part of titin is formed by both Ig and Fn3 domains.
Purified titin molecules visualized by metal shadowing appear in electron micrographs as strings about one micrometer in length and four nanometers in diameter [15-18]. In negatively stained samples, a distinct "beads-on-the-string" appearance can be seen [15], showing the chain of Ig and Fn3-like domains $[19,20]$.

\section{Titin Structure-Conformational Periodicity in the Thick Filament Region}

Sequence shows that the titin Ig and Fn3 domains are arranged in long-range patterns or super-repeats. Two types of super-repeats are found in the constitutively expressed thick filament region [19-21]: seven consecutive copies of the seven-domain or small superrepeat occupy the N-terminal part of this region; these are followed by eleven copies of the eleven-domain large superrepeat. In the I-band region, only the differentially expressed Ig-segments have superrepeats: in human soleus isoform, the N-terminal three copies of a six-domain superrepeat are followed by three copies of a ten-domain superrepeat $[22,23]$. In both A- and I-band regions, the super-repeats show increased sequence conservation between domains at comparable positions in the super-repeats.

The two super-repeats of Ig and Fn3 domains in A-band titin (Figure 1(b)) closely reflect the underlying periodic 


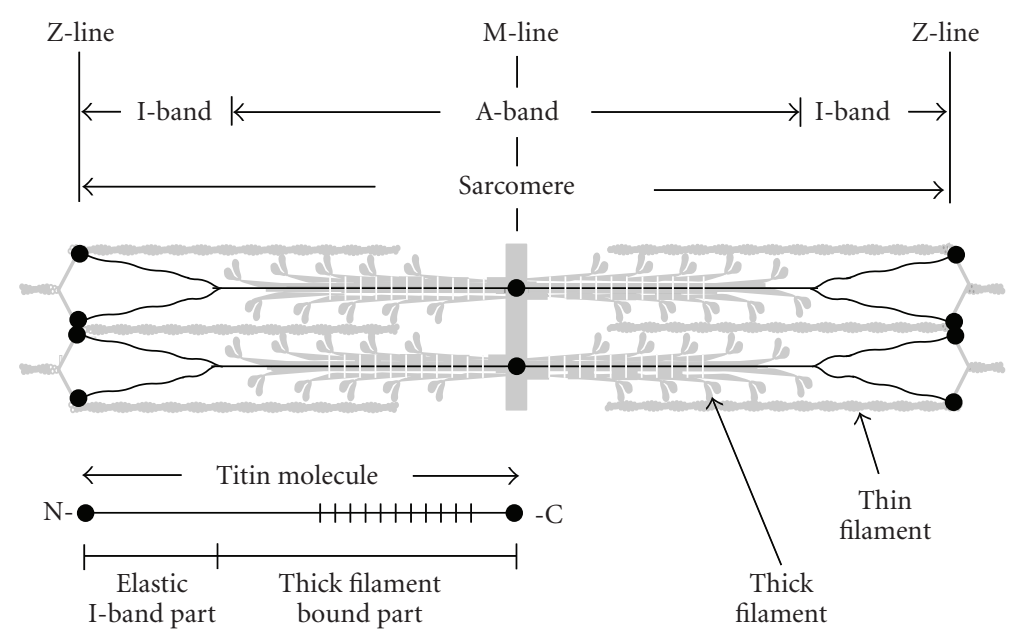

(a)

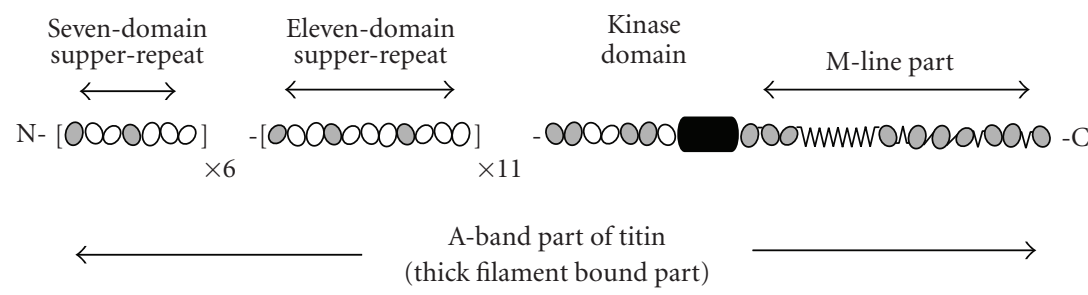

(b)

FIGURE 1: Schematic representation of titin layout in the sarcomere (a) and of the domain periodicity in the thick filament bound part (b). Striated zones in the sarcomere and the titin molecule (a) show the location of the large superrepeat.

structure of the thick filament. The size of the large superrepeat $(\sim 45 \mathrm{~nm})$ is same as the myosin and C-protein periodicities of the filament, while the number of the large super-repeats (eleven) is the same as the number of binding sites for C-protein and related molecules. A single Ig domain in each large superrepeat binds $\mathrm{C}$-protein and this defines the $\sim 43 \mathrm{~nm}$ interval of C-protein localization in the C-zone [24]. Interactions between titin and myosin are probably grouped in three clusters of Fn 3 domains, defining a period of $\sim 15 \mathrm{~nm}$ in titin-myosin interactions [19].

Atomic structures of recombinant titin fragments, supported by homology modelling $[25,26]$, indicate that the Ig and Fn3 domains are unlikely to be similarly oriented along a titin molecule but bend and twist relative to each other. The likelihood of periodic interactions with other thick filament proteins requires titin domains spaced by $43 \mathrm{~nm}$ to be in the same orientation. This indicates that in the A-band the orientation of Ig and Fn3 domains in the super-repeats is periodically repeated.

There are only two main possibilities for such periodic architecture: either planar and zigzag-like or threedimensional and helical. Homology modelling [26, 27] and crystallography studies [28, 29] show that small, twoor three-domain recombinant fragments have rather flat conformations, in which the long axes of the molecules bend in a single plane. However, longer segments may well have a tendency to bend three-dimensionally, as suggested by modelling of the periodic differentially expressed I-band Ig-segment [30]. The latter shape is supported by electron microscopy of purified titin also suggesting helicity [31]. A tendency of the relaxed titin molecule to adopt a helical conformation is likely to be controlled by a preferred interdomain orientation $[25,30,32]$ and may reflect a longdistance directional regularity in the bending and twisting angles. The existing atomic structures of titin fragments appear to be consistent with this possibility [30], although the number of these is still too small to discern a longdistance pattern.

Another important question concerning titin interactions with other thick filament proteins is the significance of the fact that super-repeats in the A-band titin, although very similar, are not identical. Average sequence identity, even for domains at comparable positions, is below $40 \%$ [27], and only about $60 \%$ of their surface is conserved [33]. It is unclear at present how these small differences affect periodic interactions of titin with C-protein and myosin, both of which provide identical sites for interaction with super-repeats. It may be noted, however, that if there are dissimilar titin-myosin and titin-C-protein interactions in different super-repeats, this would agree with predictions 
of nonequivalency of the $\sim 43 \mathrm{~nm}$ repeats in thick filament structure suggested by X-ray diffraction studies of muscle [34].

\section{Titin Flexibility-Persistence Length of the Elastic I-Band Region}

Providing elasticity to sarcomeres is one of the major functions of titin [35-39]. This role derives from the ability of titin to increase the length under applied force and then to shorten to the original length when the force is removed. The mechanism of extensibility is known to be multiphase: overall shape changes that occur under small applied forces are followed at higher forces by hierarchical unfolding of the polypeptide [40-43]. While the physiological relevance of relatively large sarcomere lengths that lead to titin unfolding can be disputed, there is no doubt that conformational changes must occur in the molecule during both passive extension and active contraction of muscle. However, the exact pattern of these changes remains unknown. It is generally thought that they are entropic in nature and occur according to rod-coil transitions seen in individual titin molecules in vitro (e.g., [31]). However, the likely bundled state of titin molecules in the sarcomere is not usually taken into account [44-48]. In the following, we present some estimates related to titin flexibility in vitro and in situ.

Electron and atomic force microscopy (AFM) of purified titin molecules illustrate a tendency to coil up in the absence of applied extensional force (Figure 2(a)) [15, 16, 31, 49] or to straighten when a small pulling force is applied $[17,18$, $31,38,50]$. The persistence length $(L p)$ of monomeric titin is estimated to be $9-19 \mathrm{~nm}[31,49,51,52]$. A somewhat smaller range of values, $2-10 \mathrm{~nm}$, was suggested by AFM and optical tweezer mechanical experiments on single molecules [43, 53$55]$, and a tendency of the protein to unfolding was discussed $[53,55]$.

The estimates of $L p$ are likely to mainly reflect average flexibility of the Ig/Fn3 parts of the molecule, since the unique sequences and other structures are a small proportion of the molecule. In a multidomain protein, overall bending flexibility mainly derives from the mobility at the interdomain interfaces. Taking average interdomain distance $s$ to be about $4.0 \mathrm{~nm}$ [27] and the average persistence length $L p$ to be about $13.5 \mathrm{~nm}$, the average inter-domain angle can be approximately estimated from the relationship $\left\langle\theta^{2}(s)\right\rangle=$ $2 s / L p$ [56], from which a $\langle\theta\rangle$ value of $\sim 44^{\circ}$ can be obtained. This value (i.e., $180^{\circ}-44^{\circ}=136^{\circ}$ ) is close to the average inter-domain angle in the NMR and crystal structures of titin fragments, $\sim 140^{\circ}[25,26,28-30,32,57,58]$. This suggests that the inter-domain angles and shapes of titin constructs reflect the equilibrium-relaxed conformation of the native titin molecule. In this conformation, the inter-domain angles and twists are likely to be different from those in situ, at least in the case of A-band titin, which is extended and stressed by the interactions with myosin and C-protein in the thick filament backbone.

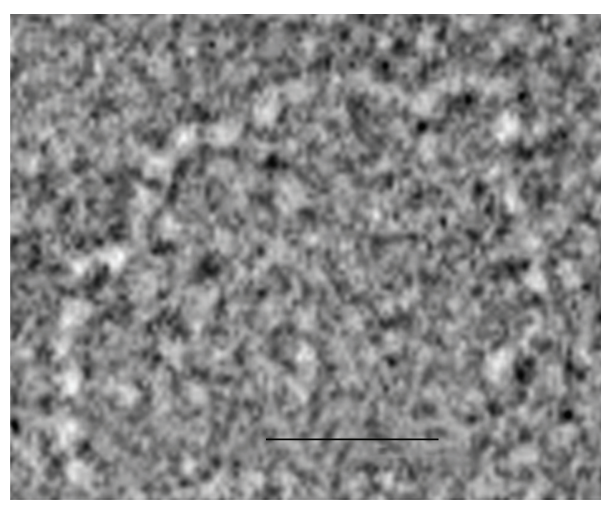

(a)

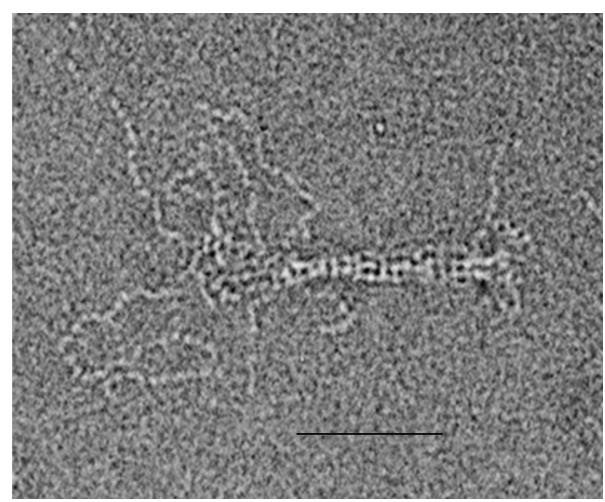

(b)

FIGURE 2: Electron micrographs of negatively stained titin, illustrating domain substructure and flexibility (a), and the effect of bundling on the apparent stiffness of the molecule (b). (a) Note that each of the flexibility "waves" seen in the titin contour contains about 4 domains. This number is comparable with the number of domains (3-4) expected for segments of about the persistence length of the protein. (b) This micrograph shows partially dissolved titin "end-filament". The "wavy" contours of the molecules in the unbundled region contrast with their relatively straight shapes in the bundled part. Magnification: bar (a) $20 \mathrm{~nm}$; (b) $50 \mathrm{~nm}$.

Only slightly higher $L p$ values $(15-40 \mathrm{~nm})$ were estimated for individual titin molecules from mechanical experiments on muscle fibres [41] and myofibrils [59]. The difference between the in vitro and in situ results is smaller than might be expected since, in the sarcomere, the extensible I-band parts of titin are known to be bundled [44-48]. Inspection of the bundled and unbundled parts of I-band titin seen in electron microscope images (Figure 2(b)) clearly illustrates different bending properties. As was estimated earlier for an analogous case [60], the persistence length of a bundle scales with the square of the number of subfilaments in the bundle, that is, $L p \sim N 2$. In the case of titin, this means that a bundle of six molecules will make $L p$ at least $500 \mathrm{~nm}$, that is, 36 times larger than $L p$ for a single molecule, taken as $13.5 \mathrm{~nm}$. Even for smaller bundles, composed of only twothree molecules, the expected $L p$ value is in the region of $\sim 55-120 \mathrm{~nm}$.

The two $L p$ values, $55-120 \mathrm{~nm}$ and $500 \mathrm{~nm}$, reflect the expected stiffness of the two Ig-tandem segments of I-band 
titin that are separated by the unique N2-PEVK region: the proximal N-terminal segment, attached to the Z-line, and the bundled distal C-terminal segment, attached to the tip of the thick filament, known as the end-filament [44-48]. The fact that the $L p$ values from mechanical experiments on muscle fibres and myofibrils closely correspond to the in vitro $L p$ value of the monomeric molecule is unlikely to correctly reflect titin's state and flexibility in situ. Alternatively, this may indicate dissociation of end-filaments during experiments, possibly due to their fragility, or may reflect the specificity of conformational changes in situ, related, for instance, to helicity (see above) and/or the confined environment in the sarcomere.

\section{Adaptation of Titin Organization in the Sarcomere to the Symmetry Mismatch between $Z$ - and $M$-Lines}

How the arrangement of titin in the sarcomere accommodates the different symmetries of thick and thin filament lattices, and of the $\mathrm{M}$ - and Z-line regions, is a major unresolved problem in sarcomere structure. The number of six molecules bound to each half of thick filament [39, 47] correlates well with the threefold rotational symmetry of the filament [61], its three-stranded substructure $[44,62$, $63]$, and with the hexagonal lattice of the $\mathrm{M}$-line region $[61,64]$. However, titin extension through the I-band, and especially its interaction with thin filaments near and within Z-line region, suggests a rearrangement to fit to the twofold rotational symmetry of thin filaments and the tetragonal lattice of the Z-line. The question is thus how titin molecules rearrange in the I-band.

One of the factors that may affect this rearrangement is self-association (see also above). Titin self-association in the I-band is suggested by electron microscope studies of muscle and separate thick filaments, which show the $\sim 100 \mathrm{~nm}$ stalk-like structures called end-filaments, referred to above, projecting from the ends of thick filaments [44-46]. It is also supported by in vitro observations of self-association of the titin segment from this region [48]. This includes the entire distal (with respect to the Z-line) tandem-Ig segment of Iband titin, from the thick filament tip up to the PEVK-N2 region. At this point the bundle is likely to branch, although not necessarily into single molecules.

The relatively high negative charge on the PEVK (N2B) region(s) of titin would favour branching of the endfilaments, unless the charge is neutralised by interactions either with cations (e.g., $\mathrm{Ca}^{++}$ions $[65,66]$ ) or with other cytoplasmic components. It should also be noted that the N2-PEVK-region is the site where thin filaments appear to rearrange from hexagonal to tetragonal packing, which then becomes especially ordered near and within the Z-line [67]. However, structural studies have so far failed to provide an unambiguous answer for the number of the branched subfilaments. Three alternatives end-filament branching schemes are possible: (1) into two subfilaments, each containing three titin molecules; (2) into three subfilaments with two titin molecules in each; and (3) into six subfilaments, each a single titin molecule. In the first case, such a division would give $1: 1$ ratio of titin to thin filaments, which would imply interaction of each thin filament with a single bundle of three titin molecules near and within the $\mathrm{Z}$-line region. This would satisfy tetragonal Z-line symmetry; however, there would be no agreement with the twofold symmetry of thin filament. Splitting the end-filament into a larger number of subfilaments would not satisfy either the Z-line or thin filament symmetries.

Thus, some asymmetry has to be assumed to exist in the titin arrangement and interactions in either the I-band or within the Z-line. A possible arrangement of titin in the Z-line has been discussed [47]. This scheme suggests splitting of end-filament into six individual titin molecules with only four of these molecules interacting side-by-side with two thin filaments of the same sarcomere and extending throughout the Z-line. The remaining two titins would attach to the tips of two incoming thin filaments of the adjacent sarcomere. This arrangement gives a $2: 1$ ratio of side-by-side interactions between titin molecules and thin filaments and is in agreement with both thin filament and Z-line symmetries. It would also provide the required mechanical balance in Z-line region and appears to be in a good agreement with structural data [68].

Another possibility relates to involvement of small titin isoforms, for example, Novex-3, which are present in skeletal and cardiac muscles in varying amounts in parallel with the main full-length isoforms, but which span only half the Iband, between the Z-line and N2-line [14, 69]. This location potentially helps to resolve the problem of correlation of the number of titin molecules with the thin filaments and Z-line symmetries. However, the low amount of Novex-3 expressed by muscles [14] is apparently not compatible with this role of the protein. Also, involvement of additional isoforms would not eliminate the asymmetry in titin interactions in the Iband but will only shift the site of asymmetry from the Zline to the N2-line region of sarcomere where the different titin isoforms meet.

\section{Concluding Remarks}

Although an enormous amount has been learnt about the properties of the titin molecule, both in vitro and in situ, integrating this information to give a comprehensive picture is not straightforward. This is mainly because in no part of the sarcomere-A- or I-band, Z- or M-lineis the disposition of the components known in molecular detail. Much therefore remains to be learnt about sarcomere structure and titin layout and function, and how these are compromised in disease.

\section{Acknowledgment}

The authors thank the British Heart Foundation for supporting this work (PG/06/006/20236). 


\section{References}

[1] S. Y. Boateng and P. H. Goldspink, "Assembly and maintenance of the sarcomere night and day," Cardiovascular Research, vol. 77, no. 4, pp. 667-675, 2008.

[2] E. Ehler and M. Gautel, "The sarcomere and sarcomerogenesis," Advances in Experimental Medicine and Biology, vol. 642, pp. 1-14, 2008.

[3] M. S. Willis, J. C. Schisler, A. L. Portbury, and C. Patterson, "Build it up-tear it down: protein quality control in the cardiac sarcomere," Cardiovascular Research, vol. 81, no. 3, pp. 439448, 2009.

[4] W. G. Pyle and R. J. Solaro, "At the crossroads of myocardial signaling: the role of Z-discs in intracellular signaling and cardiac function," Circulation Research, vol. 94, no. 3, pp. 296305, 2004.

[5] R. J. Solaro and P. P. De Tombe, "Review focus series: sarcomeric proteins as key elements in integrated control of cardiac function," Cardiovascular Research, vol. 77, no. 4, pp. 616-618, 2008.

[6] L. Tskhovrebova and J. Trinick, "Titin: properties and family relationships," Nature Reviews Molecular Cell Biology, vol. 4, no. 9, pp. 679-689, 2003.

[7] H. Granzier and S. Labeit, "Structure-function relations of the giant elastic protein titin in striated and smooth muscle cells," Muscle and Nerve, vol. 36, no. 6, pp. 740-755, 2007.

[8] W. A. Linke, "Sense and stretchability: the role of titin and titin-associated proteins in myocardial stress-sensing and mechanical dysfunction," Cardiovascular Research, vol. 77, no. 4, pp. 637-648, 2008.

[9] M. L. Greaser, "Stressing the giant: a new approach to understanding dilated cardiomyopathy," Journal of Molecular and Cellular Cardiology, vol. 47, no. 3, pp. 347-349, 2009.

[10] A. Kontrogianni-Konstantopoulos, M. A. Ackermann, A. L. Bowman, S. V. Yap, and R. J. Bloch, "Muscle giants: molecular scaffolds in sarcomerogenesis," Physiological Reviews, vol. 89, no. 4, pp. 1217-1267, 2009.

[11] K. Maruyama, T. Yoshioka, and H. Higuchi, "Connectin filaments link thick filaments and $\mathrm{Z}$ lines in frog skeletal muscle as revealed by immunoelectron microscopy," Journal of Cell Biology, vol. 101, no. 6, pp. 2167-2172, 1985.

[12] D. O. Furst, M. Osborn, R. Nave, and K. Weber, "The organization of titin filaments in the half-sarcomere revealed by monoclonal antibodies in immunoelectron microscopy: a map of ten nonrepetitive epitopes starting at the $\mathrm{Z}$ line extends close to the M line," Journal of Cell Biology, vol. 106, no. 5, pp. 1563-1572, 1988.

[13] D. O. Fürst, R. Nave, M. Osborn, and K. Weber, "Repetitive titin epitopes with a $42 \mathrm{~nm}$ spacing coincide in relative position with known A band striations also identified by major myosin-associated proteins. An immunoelectronmicroscopical study on myofibrils," Journal of cell science, vol. 94, pp. 119-125, 1989.

[14] M.-L. Bang, T. Centner, F. Fornoff et al., "The complete gene sequence of titin, expression of an unusual $\approx 700-\mathrm{kDa}$ titin isoform, and its interaction with obscurin identify a novel Zline to I-band linking system," Circulation Research, vol. 89, no. 11, pp. 1065-1072, 2001.

[15] J. Trinick, P. Knight, and A. Whiting, "Purification and properties of native titin," Journal of Molecular Biology, vol. 180, no. 2, pp. 331-356, 1984.

[16] K. Wang, R. Ramirez-Mitchell, and D. Palter, "Titin is an extraordinarily long, flexible, and slender myofibrillar protein," Proceedings of the National Academy of Sciences of the United States of America, vol. 81, no. 12, pp. 3685-3689, 1984.

[17] R. Nave, D. O. Furst, and K. Weber, "Visualization of the polarity of isolated titin molecules: a single globular head on a long thin rod as the M band anchoring domain?" Journal of Cell Biology, vol. 109, no. 5, pp. 2177-2187, 1989.

[18] M. Sonoda, S. Kimura, H. Moriya, Y. Shimada, and K. Maruyama, "Molecular shape of $\alpha$-Connectin, an elastic filamentous protein of skeletal muscle," Proceedings of the Japan Academy. Series B, vol. 66, no. 10, pp. 213-216, 1990.

[19] S. Labeit, M. Gautel, A. Lakey, and J. Trinick, "Towards a molecular understanding of titin," EMBO Journal, vol. 11, no. 5, pp. 1711-1716, 1992.

[20] S. Labeit and B. Kolmerer, "Titins: giant proteins in charge of muscle ultrastructure and elasticity," Science, vol. 270, no. 5234, pp. 293-296, 1995.

[21] S. Labeit, D. P. Barlow, M. Gautel et al., "A regular pattern of two types of 100-residue motif in the sequence of titin," Nature, vol. 345, no. 6272, pp. 273-276, 1990.

[22] M. Gautel, "The super-repeats of titin/connectin and their interactions: glimpses at sarcomeric assembly," Advances in Biophysics, vol. 33, pp. 27-37, 1996.

[23] C. C. Witt, N. Olivieri, T. Centner et al., "A survey of the primary structure and the interspecies conservation of I-band titin's elastic elements in vertebrates," Journal of Structural Biology, vol. 122, no. 1-2, pp. 206-215, 1998.

[24] A. Freiburg and M. Gautel, "A molecular map of the interactions between titin and myosin-binding protein $\mathrm{C}$. Implications for sarcomeric assembly in familial hypertrophic cardiomyopathy," European Journal of Biochemistry, vol. 235, no. 1-2, pp. 317-323, 1996.

[25] S. Improta, J. K. Krueger, M. Gautel et al., "The assembly of immunoglobulin-like modules in titin: implications for muscle elasticity," Journal of Molecular Biology, vol. 284, no. 3, pp. 761-777, 1998.

[26] C. Muhle-Goll, A. Pastore, and M. Nilges, "The threedimensional structure of a type I module from titin: a prototype of intracellular fibronectin type III domains," Structure, vol. 6, no. 10, pp. 1291-1302, 1998.

[27] P. Amodeo, F. Fraternali, A. M. Lesk, and A. Pastore, "Modularity and homology: modelling of the titin type I modules and their interfaces," Journal of Molecular Biology, vol. 311, no. 2, pp. 283-296, 2001.

[28] M. Mrosek, D. Labeit, S. Witt et al., "Molecular determinants for the recruitment of the ubiquitin-ligase MuRF-1 onto Mline titin," FASEB Journal, vol. 21, no. 7, pp. 1383-1392, 2007.

[29] S. Müller, S. Lange, M. Gautel, and M. Wilmanns, "Rigid conformation of an immunoglobulin domain tandem repeat in the A-band of the elastic muscle protein titin," Journal of Molecular Biology, vol. 371, no. 2, pp. 469-480, 2007.

[30] E. Von Castelmur, M. Marino, D. I. Svergun et al., "A regular pattern of Ig super-motifs defines segmental flexibility as the elastic mechanism of the titin chain," Proceedings of the National Academy of Sciences of the United States of America, vol. 105, no. 4, pp. 1186-1191, 2008.

[31] L. Tskhovrebova and J. Trinick, "Flexibility and extensibility in the titin molecule: analysis of electron microscope data," Journal of Molecular Biology, vol. 310, no. 4, pp. 755-771, 2001.

[32] M. Marino, P. Zou, D. Svergun et al., “The Ig Doublet Z1Z2: a model system for the hybrid analysis of conformational dynamics in Ig tandems from titin," Structure, vol. 14, no. 9, pp. 1437-1447, 2006. 
[33] C. Muhle-Goll, M. Habeck, O. Cazorla, M. Nilges, S. Labeit, and H. Granzier, "Structural and functional studies of titin's fn 3 modules reveal conserved surface patterns and binding to myosin S1-a possible role in the Frank-Starling mechanism of the heart," Journal of Molecular Biology, vol. 313, no. 2, pp. 431-447, 2001.

[34] J. M. Squire, M. Roessle, and C. Knupp, "New X-ray diffraction observations on vertebrate muscle: organisation of Cprotein (MyBP-C) and troponin and evidence for unknown structures in the vertebrate A-band," Journal of Molecular Biology, vol. 343, no. 5, pp. 1345-1363, 2004.

[35] K. Maruyama, T. Yoshioka, and H. Higuchi, "Connectin filaments link thick filaments and $\mathrm{Z}$ lines in frog skeletal muscle as revealed by immunoelectron microscopy," Journal of Cell Biology, vol. 101, no. 6, pp. 2167-2172, 1985.

[36] K. Wang, "Sarcomere-associated cytoskeletal lattices in striated muscle. Review and hypothesis," Cell and Muscle Motility, vol. 6, pp. 315-369, 1985.

[37] R. Horrowits and R. J. Podolsky, "The positional stability of thick filaments in activated skeletal muscle depends on sarcomere length: evidence for the role of titin filaments," Journal of Cell Biology, vol. 105, no. 5, pp. 2217-2223, 1987.

[38] A. Soteriou, A. Clarke, S. Martin, and J. Trinick, "Titin folding energy and elasticity," Proceedings of the Royal Society B, vol. 254, no. 1340, pp. 83-86, 1993.

[39] H. L. Granzier and T. C. Irving, "Passive tension in cardiac muscle: contribution of collagen, titin, microtubules, and intermediate filaments," Biophysical Journal, vol. 68, no. 3, pp. 1027-1044, 1995.

[40] M. Gautel and D. Goulding, "A molecular map of titin/connectin elasticity reveals two different mechanisms acting in series," FEBS Letters, vol. 385, no. 1-2, pp. 11-14, 1996.

[41] K. Trombitás, M. Greaser, S. Labeit et al., "Titin extensibility in situ: entropic elasticity of permanently folded and permanently unfolded molecular segments," Journal of Cell Biology, vol. 140, no. 4, pp. 853-859, 1998.

[42] K. Trombitás, A. Redkar, T. Centner, Y. Wu, S. Labeit, and H. Granzier, "Extensibility of isoforms of cardiac titin: variation in contour length of molecular subsegments provides a basis for cellular passive stiffness diversity," Biophysical Journal, vol. 79, no. 6, pp. 3226-3234, 2000.

[43] H. Li, W. A. Linke, A. F. Oberhauser et al., "Reverse engineering of the giant muscle protein titin," Nature, vol. 418, no. 6901, pp. 998-1002, 2002.

[44] J. A. Trinick, "End filaments: a new structural element of vertebrate skeletal muscle thick filaments," Journal of Molecular Biology, vol. 151, no. 2, pp. 309-314, 1981.

[45] T. Funatsu, E. Kono, H. Higuchi et al., "Elastic filaments in situ in cardiac muscle: deep-etch replica analysis in combination with selective removal of actin and myosin filaments," Journal of Cell Biology, vol. 120, no. 3, pp. 711-724, 1993.

[46] P. M. Bennett, T. E. Hodkin, and C. Hawkins, "Evidence that the tandem ig domains near the end of the muscle thick filament form an inelastic part of the I-band titin," Journal of Structural Biology, vol. 120, no. 1, pp. 93-104, 1997.

[47] A. D. Liversage, D. Holmes, P. J. Knight, L. Tskhovrebova, and J. Trinick, "Titin and the sarcomere symmetry paradox," Journal of Molecular Biology, vol. 305, no. 3, pp. 401-409, 2001.

[48] A. Houmeida, A. Baron, J. Keen et al., "Evidence for the oligomeric state of 'elastic' titin in muscle sarcomeres," Journal of Molecular Biology, vol. 384, no. 2, pp. 299-312, 2008.
[49] M. S. Z. Kellermayer, C. Bustamante, and H. L. Granzier, "Mechanics and structure of titin oligomers explored with atomic force microscopy," Biochimica et Biophysica Acta, vol. 1604, no. 2, pp. 105-114, 2003.

[50] L. Tskhovrebova and J. Trinick, "Direct visualization of extensibility in isolated titin molecules," Journal of Molecular Biology, vol. 265, no. 2, pp. 100-106, 1997.

[51] H. Higuchi, Y. Nakauchi, K. Maruyama, and S. Fujime, "Characterization of $\beta$-connectin (titin 2) from striated mucle by dynamic light scattering," Biophysical Journal, vol. 65, no. 5, pp. 1906-1915, 1993.

[52] E. Di Cola, T. A. Waigh, J. Trinick et al., "Persistence length of titin from rabbit skeletal muscles measured with scattering and microrheology techniques," Biophysical Journal, vol. 88, no. 6 , pp. 4095-4106, 2005.

[53] M. S. Z. Kellermayer, S. B. Smith, H. L. Granzier, and C. Bustamante, "Folding-unfolding transitions in single titin molecules characterized with laser tweezers," Science, vol. 276, no. 5315, pp. 1112-1116, 1997.

[54] M. Rief, M. Gautel, F. Oesterhelt, J. M. Fernandez, and H. E. Gaub, "Reversible unfolding of individual titin immunoglobulin domains by AFM," Science, vol. 276, no. 5315, pp. 11091112, 1997.

[55] L. Tskhovrebova, J. Trinick, J. A. Sleep, and R. M. Simmons, "Elasticity and unfolding of single molecules of the giant muscle protein titin," Nature, vol. 387, no. 6630, pp. 308-312, 1997.

[56] A. Yu. Grosberg and A. R. Khokhlov, "Statistical Physics of Macromolecules, AIP Press, New York, NY, USA, 2004.

[57] M. Marino, D. I. Svergun, L. Kreplak et al., "Poly-Ig tandems from I-band titin share extended domain arrangements irrespective of the distinct features of their modular constituents," Journal of Muscle Research and Cell Motility, vol. 26, no. 6-8, pp. 355-365, 2005.

[58] P. Zou, N. Pinotsis, S. Lange et al., "Palindromic assembly of the giant muscle protein titin in the sarcomeric Z-disk," Nature, vol. 439, no. 7073, pp. 229-233, 2006.

[59] W. A. Linke, M. R. Stockmeier, M. Ivemeyer, H. Hosser, and P. Mundel, "Characterizing titin's I-band Ig domain region as an entropic spring," Journal of Cell Science, vol. 111, no. 11, pp. 1567-1574, 1998.

[60] J. C. Wang, M. S. Turner, G. Agarwal et al., "Micromechanics of isolated sickle cell hemoglobin fibers: bending moduli and persistence lengths," Journal of Molecular Biology, vol. 315, no. 4, pp. 601-612, 2002.

[61] P. K. Luther, P. M. G. Munro, and J. M. Squire, "Threedimensional structure of the vertebrate muscle A-band. III. M-region structure and myosin filament symmetry," Journal of Molecular Biology, vol. 151, no. 4, pp. 703-730, 1981.

[62] M. C. Maw and A. J. Rowe, "Fraying of a-filaments into three subfilaments," Nature, vol. 286, no. 5771, pp. 412-414, 1980.

[63] R. W. Kensler and M. Stewart, "Frog skeletal muscle thick filaments are three-stranded," Journal of Cell Biology, vol. 96, no. 6, pp. 1797-1802, 1983.

[64] G. G. Knappeis and F. Carlsen, "The ultrastructure of the M line in skeletal muscle," Journal of Cell Biology, vol. 38, no. 1, pp. 202-211, 1968.

[65] R. Tatsumi, K. Maeda, A. Hattori, and K. Takahashi, "Calcium binding to an elastic portion of connectin/titin filaments," Journal of Muscle Research and Cell Motility, vol. 22, no. 2, pp. 149-162, 2001. 
[66] D. Labeit, K. Watanabe, C. Witt et al., "Calcium-dependent molecular spring elements in the giant protein titin," Proceedings of the National Academy of Sciences of the United States of America, vol. 100, no. 23, pp. 13716-13721, 2003.

[67] L. Traeger, J. M. Mackenzie Jr., H. F. Epstein, and M. A. Goldstein, "Transition in the thin-filament arrangement in rat skeletal muscle," Journal of Muscle Research and Cell Motility, vol. 4, no. 3, pp. 353-366, 1983.

[68] C. Knupp, P. K. Luther, and J. M. Squire, “Titin organisation and the 3D architecture of the vertebrate-striated muscle Iband," Journal of Molecular Biology, vol. 322, no. 4, pp. 731739, 2002.

[69] H. Granzier, M. Radke, J. Royal et al., "Functional genomics of chicken, mouse, and human titin supports splice diversity as an important mechanism for regulating biomechanics of striated muscle," American Journal of Physiology, vol. 293, no. 2, pp. R557-R567, 2007. 

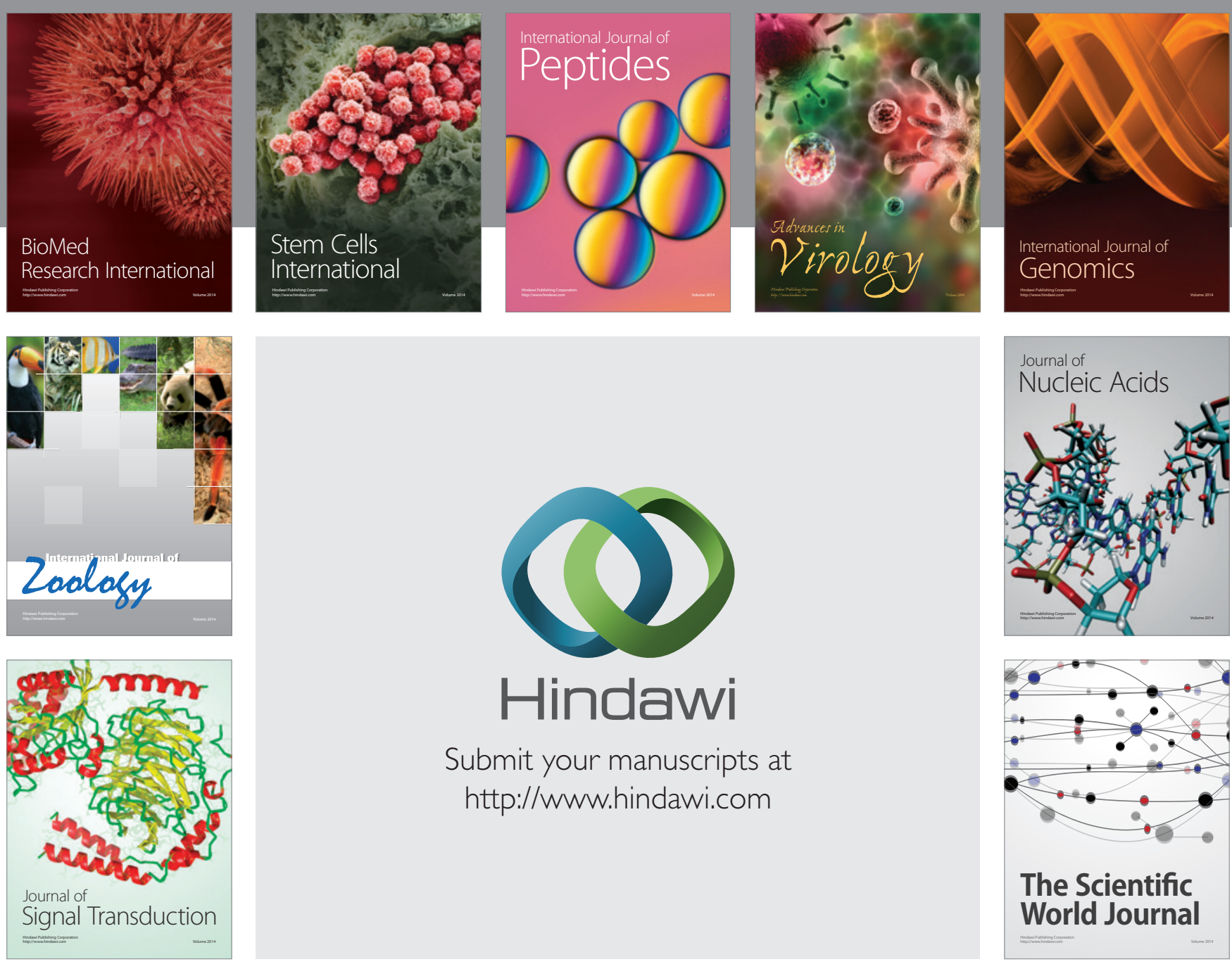

Submit your manuscripts at

http://www.hindawi.com
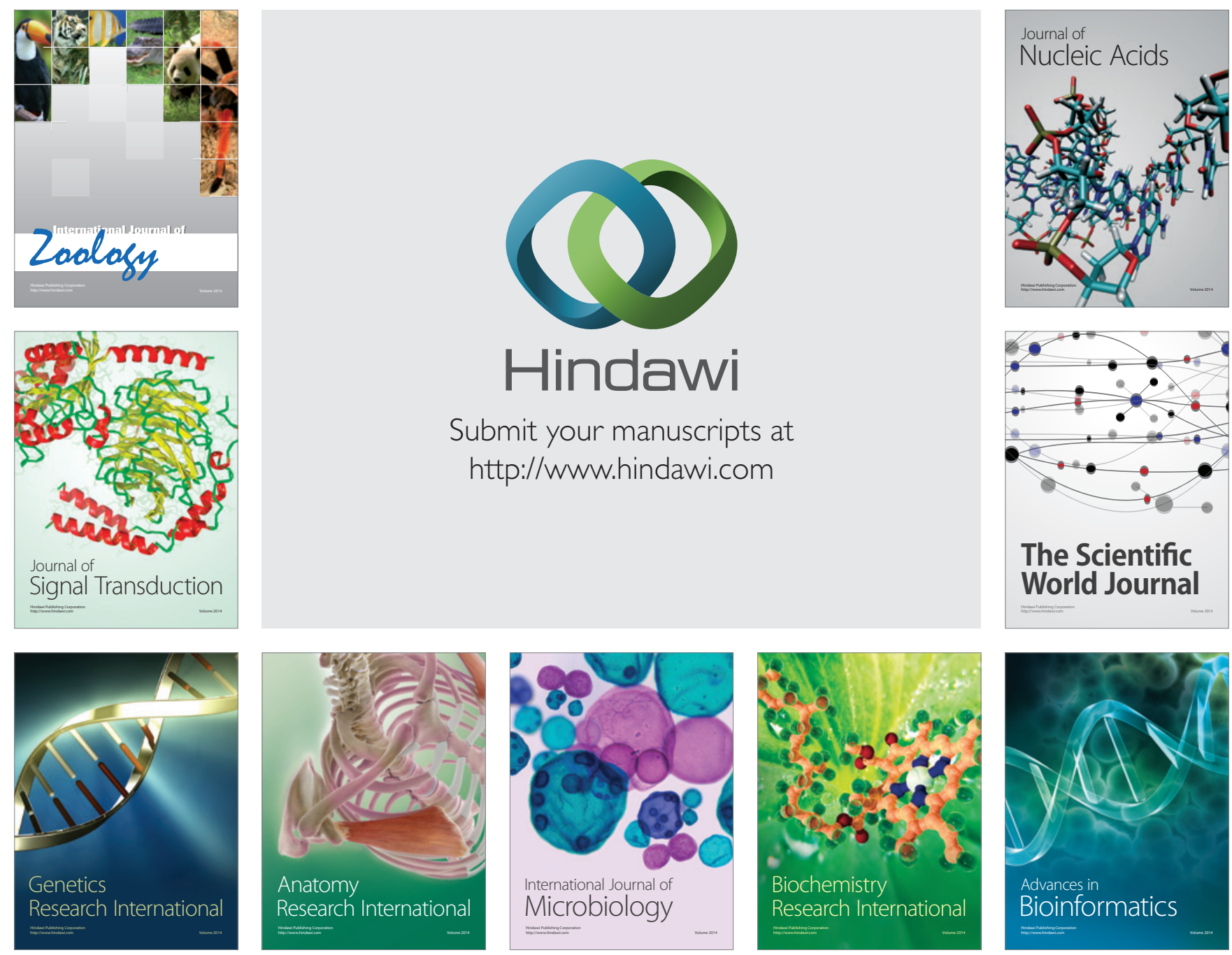

The Scientific World Journal
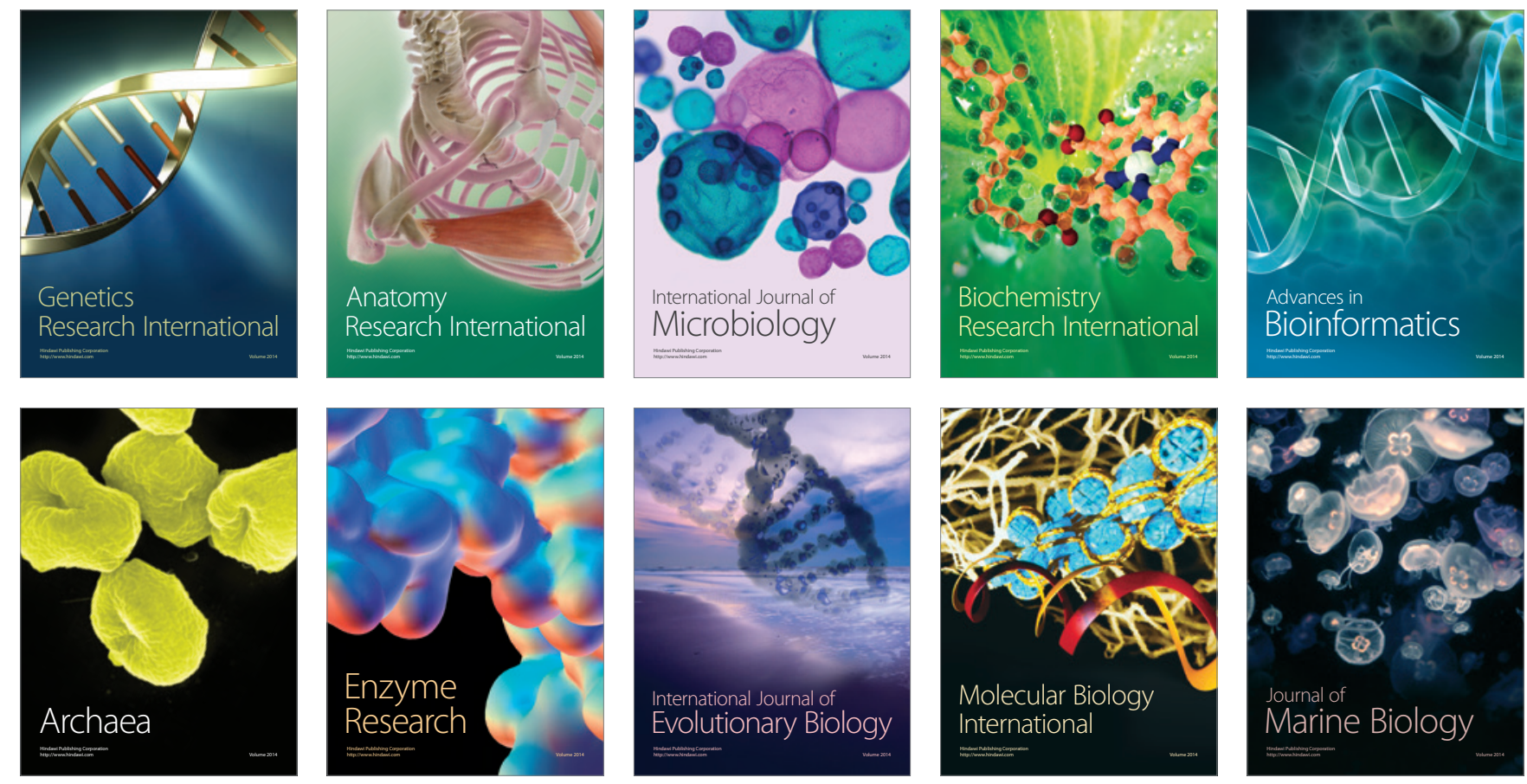